\title{
EIGENFUNCTIONS OF THE LAPLACE-BELTRAMI OPERATOR ON HYPERBOLOIDS
}

\author{
AMRITANSHU PRASAD AND M. K. VEMURI
}

\begin{abstract}
Eigenfunctions of the Laplace-Beltrami operator on a hyperboloid are studied in the spirit of the treatment of the spherical harmonics by Stein and Weiss. As a special case, a simple self-contained proof of Laplace's integral for a Legendre function is obtained.
\end{abstract}

In [2, Chapter IV, Section 2], Stein and Weiss described the spectral decomposition of the Laplace-Beltrami operator on the unit sphere. Their approach was to identify the eigenfunctions with homogeneous harmonic functions on Euclidean space.

In this article the eigenfunctions of the Laplace-Beltrami operator on a hyperboloid are identified with homogeneous harmonic functions (with respect to a Laplacian of type $(p, q)$ ) on an open cone. In the case treated by Stein and Weiss, Liouville's theorem implies that the degree of homogeneity must be a non-negative integer, whereas here the degree of homogeneity can be any complex number. This identification is used to compute spherical functions for $O(1, q)$, and consequently Laplace's integral formula for Legendre functions is obtained. Laplace's integral formula can also be obtained by using the residue theorem [3, \$15.23]. Spherical functions for semisimple Lie groups in general are obtained using different methods (see, e.g., [1, Chapter IV]).

Let $n=p+q$. Let $\mathbf{R}^{p, q}$ denote the space of real $n$-dimensional vectors equipped with the indefinite scalar product of signature $(p, q)$ :

$$
\mathbf{x} \cdot \mathbf{y}={ }^{t} \mathbf{x} Q \mathbf{y}
$$

where $Q$ is the diagonal matrix with $p$ 1's followed by $q(-1)$ 's along the diagonal. Write $|\mathbf{x}|^{2}$ for $\mathbf{x} \cdot \mathbf{x}$. There should be no confusion with the usual positive definite dot product and norm as they are never used in this paper.

Let $\mathbf{R}_{+}^{p, q}$ denote the subset of $\mathbf{R}^{p, q}$ consisting of those vectors for which $|\mathbf{x}|^{2}>0$. For $\mathbf{x} \in$ $\mathbf{R}_{+}^{p, q}$, let $|\mathbf{x}|$ denote the positive square root of $|\mathbf{x}|^{2}$. Let $O(p, q)$ denote the group consisting of matrices such that ${ }^{t} A Q A=Q$. Denote by $O(p, q)_{0}$ the connected component of the identity element of $O(p, q)$. Let $S^{p, q}$ denote the connected component of $(1,0, \ldots, 0)$ in the hyperboloid

$$
\left\{\mathbf{x}:|\mathbf{x}|=1, x_{1}>0\right\} .
$$

Received May 13, 2008.

2000 Mathematics Subject Classification. 33C55, 43A90

Key words and phrases. spherical functions, indefinite orthogonal group. 
Let $\rho$ be any complex number. Let $\mathscr{P}_{\rho}$ denote the space of all functions $f \in C^{2}\left(\mathbf{R}_{+}^{p, q}\right)$ which are homogeneous of degree $\rho$, i.e., functions such that

$$
f(\lambda \mathbf{x})=\lambda^{\rho} f(\mathbf{x}) \text { for all } \mathbf{x} \in \mathbf{R}_{+}^{p, q}, \lambda>0 .
$$

Denote by $\Delta$ the differential operator $|\nabla|^{2}=\nabla \cdot \nabla$ (using the indefinite dot product), where $\nabla$ is the gradient operator

$$
\nabla=\left(\frac{\partial}{\partial x_{1}}, \ldots, \frac{\partial}{\partial x_{n}}\right)
$$

Thus,

$$
\Delta=\left(\frac{\partial^{2}}{\partial x_{1}^{2}}+\cdots+\frac{\partial^{2}}{\partial x_{p}^{2}}\right)-\left(\frac{\partial^{2}}{\partial x_{p+1}^{2}}+\cdots+\frac{\partial^{2}}{\partial x_{n}^{2}}\right)
$$

Define

$$
\mathscr{H}_{\rho}=\left\{f \in \mathscr{P}_{\rho}: \Delta f=0\right\} .
$$

A function $u \in C^{2}\left(S^{p, q}\right)$ is called a spherical harmonic ${ }^{1}$ of degree $\rho$ if $u$ is the restriction to $S^{p, q}$ of a function in $\mathscr{H}_{\rho}$. Let $H_{\rho}$ denote the space of spherical harmonics of degree $\rho$ :

$$
H_{\rho}=\left\{\left.f\right|_{S^{p, q}}: f \in \mathscr{H}_{\rho}\right\} .
$$

The Laplace-Beltrami operator $\Delta_{S} p, q$ on $S^{p, q}$ is defined by

$$
\Delta_{S^{p}, q} u=\left.\Delta \tilde{u}\right|_{S^{p, q}}
$$

where $\tilde{u}: \mathbf{R}_{+}^{p, q} \rightarrow \mathbf{C}$ is defined by $\tilde{u}(\mathbf{x})=u(\mathbf{x} /|\mathbf{x}|$ ) (the degree zero homogeneous extension of $u$ ).

Let $\mathbf{x}^{\#}=Q \mathbf{x}$. The following is easily verified:

Lemma 1. Let $\mathbf{x} \in \mathbf{R}_{+}^{p, q}$. Then

$$
\begin{aligned}
& \nabla|\mathbf{x}|=\mathbf{x}^{\#} /|\mathbf{x}| . \\
& \nabla|\mathbf{x}|^{\rho}=\rho|\mathbf{x}|^{\rho-2} \mathbf{x}^{\#} . \\
& \left|\mathbf{x}^{\#}\right|=|\mathbf{x}| . \\
& \mathbf{x}^{\#} \cdot \nabla \tilde{u}(\mathbf{x})=0 \text { for any } u \in C^{1}\left(S^{p, q}\right) . \\
& \nabla \cdot \mathbf{x}^{\#}=n .
\end{aligned}
$$

Lemma 2. If $u \in H_{\rho}$ then $\Delta_{S^{p}, q} u=-\rho(\rho+n-2) u$.

Proof. Since $u \in H_{\rho},|\mathbf{x}|^{\rho} \tilde{u}(\mathbf{x}) \in \mathscr{\ell}_{\rho}$. Therefore (using the formulas in Lemma 1),

$$
\begin{aligned}
0 & =\Delta\left(|\mathbf{x}|^{\rho} \tilde{u}(\mathbf{x})\right) \\
& =\nabla \cdot\left(\nabla\left(|\mathbf{x}|^{\rho} \tilde{u}(\mathbf{x})\right)\right) \\
& =\nabla \cdot\left(\rho|\mathbf{x}|^{\rho-2} \mathbf{x}^{\#} \tilde{u}(\mathbf{x})+|\mathbf{x}|^{\rho} \nabla \tilde{u}(\mathbf{x})\right)
\end{aligned}
$$

\footnotetext{
${ }^{1}$ Perhaps a more apt name would be hyperboloidal harmonic.
} 


$$
\begin{aligned}
& =\left(\nabla\left(\rho|\mathbf{x}|^{\rho-2} \tilde{u}(\mathbf{x})\right) \cdot \mathbf{x}^{\#}+\rho|\mathbf{x}|^{\rho-2} \tilde{u}(\mathbf{x})\left(\nabla \cdot \mathbf{x}^{\#}\right)+|\mathbf{x}|^{\rho} \Delta \tilde{u}(\mathbf{x})\right. \\
& =\rho(\rho-2)|\mathbf{x}|^{\rho-4} \tilde{u}(\mathbf{x})\left|\mathbf{x}^{\#}\right|^{2}+\rho|\mathbf{x}|^{\rho-2} \nabla \tilde{u}(\mathbf{x}) \cdot \mathbf{x}^{\#}+n \rho|\mathbf{x}|^{\rho-2} \tilde{u}(\mathbf{x})+\Delta \tilde{u}(\mathbf{x}) \\
& =\rho(\rho-2)|\mathbf{x}|^{\rho-4} \tilde{u}(\mathbf{x})|\mathbf{x}|^{2}+n \rho|\mathbf{x}|^{\rho-2} \tilde{u}(\mathbf{x})+\Delta \tilde{u}(\mathbf{x}) .
\end{aligned}
$$

Setting $|\mathbf{x}|=1$ in the result of the above calculation yields

$$
0=\rho(\rho-2+n) \tilde{u}(\mathbf{x})+\Delta \tilde{u}(\mathbf{x}),
$$

from which the lemma follows.

The following proposition gives a construction of spherical harmonics when $p=1$ :

Proposition 3. Suppose $\mathbf{c} \in \mathbf{R}_{+}^{1, q}$ is an isotropic vector, (meaning that $|\mathbf{c}|^{2}=0$ ) such that $c_{1}>0$. Then $\mathbf{c} \cdot \mathbf{x}>0$ for all $\mathbf{x} \in S^{1, q}$. Let $f(\mathbf{x})=(\mathbf{c} \cdot \mathbf{x})^{\rho}$. Then $f \in \mathscr{H}_{\rho}$.

Proof. The set of points where $\mathbf{c} \cdot \mathbf{x}=0$ form a hyperplane tangential to the cone $|\mathbf{c}|^{2}=0$. For fixed $\mathbf{x}$, the sign of $\mathbf{c} \cdot \mathbf{x}$ can change only when $\mathbf{c}$ crosses this hyperplane. However, the entire half-cone

$$
\left\{\mathbf{c}:|\mathbf{c}|^{2}=0, \quad c_{1}>0\right\}
$$

lies on one side of the hyperplane, because the cone is quadratic. Therefore, for each $\mathbf{x} \in S^{1, q}$, it suffices to verify that $\mathbf{c} \cdot \mathbf{x}>0$ for $\mathbf{c}=(1,1,0, \ldots, 0)$. In this case, $\mathbf{c} \cdot \mathbf{x}=x_{1}-x_{2}$, which is positive since $x_{1}>0$ and $x_{1}^{2}-x_{2}^{2}-\cdots-x_{n}^{2}=1$, so that $x_{1}>\left|x_{i}\right|$ for each $i>1$.

If $g \in C^{2}\left(\mathbf{R}_{+}^{p, q}\right)$ and $\phi \in C^{2}(\mathbf{R})$, then

$$
\Delta(\phi \circ g)(\mathbf{x})=\phi^{\prime \prime}(g(\mathbf{x}))|\nabla g(\mathbf{x})|^{2}+\phi^{\prime}(g(\mathbf{x})) \Delta g(\mathbf{x}) .
$$

Let $g(\mathbf{x})=\mathbf{c} \cdot \mathbf{x}$, then $\nabla g(\mathbf{x})=\mathbf{c}$, so that $|\nabla g(\mathbf{x})|^{2}=0$. Since $g$ is linear, $\Delta g(\mathbf{x})=0$. Therefore $\Delta f(\mathbf{x})=0$.

Let $\mathbf{e}=(1,0, \ldots, 0)$. Then $K=\operatorname{Stab}_{O(1, q)_{0}}(\mathbf{e})$ is isomorphic to $S O(q)$ and is a maximal compact subgroup of $O(1, q)_{0}$. The action of $O(1, q)_{0}$ on $S^{1, q}$ is transitive, and the $K$-invariant spherical harmonics on $S^{1, q}$ are precisely the $K$-invariant spherical functions for $O(1, q)_{0}$. It follows from Proposition 3 that

Proposition 4. Let $\mathbf{c}$ be any isotropic vector in $\mathbf{R}^{1, q}$. Then

$$
\int_{K}(k \mathbf{c} \cdot \mathbf{x})^{\rho} d k
$$

is a K-invariant spherical harmonic of degree $\rho$ on $S^{1, q}$.

Since $K$ acts transitively on the slices of $S^{1, q}$ by the hyperplanes on which the first coordinate $x_{1}$ is constant, the value of a $K$-invariant spherical harmonic is simply a function of $x_{1}$, which will be denoted by $P\left(x_{1}\right)$. A $K$-invariant spherical harmonic may be viewed as a solution to an ordinary differential equation in $x_{1}$ : 
Theorem 5. Suppose that $P_{\rho}\left(x_{1}\right)$ is the value of a $K$-invariant spherical harmonic which is homogeneous of degree $\rho$. Then $P_{\rho}$ is a solution to the differential equation

$$
\left(1-x_{1}^{2}\right) P_{\rho}^{\prime \prime}\left(x_{1}\right)+(1-n) x_{1} P^{\prime}\left(x_{1}\right)+\rho(\rho-2+n) P\left(x_{1}\right)=0 .
$$

Proof. For any $f \in C^{2}\left(S^{1, q}\right)$ we have

$$
\begin{aligned}
\nabla f\left(x_{1} /|\mathbf{x}|\right) & =\nabla\left(x_{1} /|\mathbf{x}|\right) f^{\prime}\left(x_{1} /|\mathbf{x}|\right) \\
& =\frac{\left(\nabla x_{1}\right)|\mathbf{x}|-x_{1} \nabla|\mathbf{x}|}{|\mathbf{x}|^{2}} f^{\prime}\left(x_{1} /|\mathbf{x}|\right) \\
& =\frac{\mathbf{e}|\mathbf{x}|-\left(x_{1} /|\mathbf{x}|\right) \mathbf{x}^{\#}}{|\mathbf{x}|^{2}} f^{\prime}\left(x_{1} /|\mathbf{x}|\right) \\
& =u \mathbf{v},
\end{aligned}
$$

where $u=|\mathbf{x}|^{-3} f^{\prime}\left(x_{1} /|\mathbf{x}|\right)$ and $\mathbf{v}=\mathbf{e}|\mathbf{x}|^{2}-x_{1} \mathbf{x}^{\#}$. Since $\Delta=|\nabla|^{2}$,

$$
\Delta f\left(x_{1} /|\mathbf{x}|\right)=(\nabla u) \cdot \mathbf{v}+u \nabla \cdot \mathbf{v}
$$

Now,

$$
\begin{aligned}
\nabla u & =-3|\mathbf{x}|^{-5} \mathbf{x}^{\#} f^{\prime}\left(x_{1} /|\mathbf{x}|\right)+|\mathbf{x}|^{-3} \nabla\left(x_{1} /|\mathbf{x}|\right) f^{\prime \prime}\left(x_{1} /|\mathbf{x}|\right) \\
& =-3|\mathbf{x}|^{-5} \mathbf{x}^{\#} f^{\prime}\left(x_{1} /|\mathbf{x}|\right)+|\mathbf{x}|^{-6}\left(\mathbf{e}|\mathbf{x}|^{2}-x_{1} \mathbf{x}^{\#}\right) f^{\prime \prime}\left(x_{1} /|\mathbf{x}|\right) .
\end{aligned}
$$

and

$$
\nabla \cdot \mathbf{v}=\mathbf{e} \cdot \nabla|\mathbf{x}|^{2}-\left(\mathbf{e} \cdot \mathbf{x}^{\#}+n x_{1}\right)=(1-n) x_{1} .
$$

Suppose there exists a function $P$ such that $P\left(x_{1}\right)=f(\mathbf{x})$ for each $\mathbf{x}$ such that $|\mathbf{x}|=1$. Substituting the above values of $\nabla u$ and $\nabla \cdot \mathbf{v}$ in (7) and then setting $|\mathbf{x}|=1$ we have,

$$
\begin{aligned}
\left.\Delta_{S^{1, q}} f\right|_{S^{1, q}}(\mathbf{x})= & \left(-3 \mathbf{x}^{\#} P^{\prime}\left(x_{1}\right)+\left(\mathbf{e}-x_{1} \mathbf{x}^{\#}\right) P^{\prime \prime}\left(x_{1}\right)\right) \cdot\left(\mathbf{e}-x_{1} \mathbf{x}^{\#}\right) \\
& +(1-n) x_{1} P^{\prime}\left(x_{1}\right) .
\end{aligned}
$$

When $|\mathbf{x}|=1,\left|\mathbf{e}-x_{1} \mathbf{x}^{\#}\right|^{2}=\left(1-x_{1}^{2}\right)$ and $\left(\mathbf{e}-x_{1} \mathbf{x}^{\#}\right) \cdot \mathbf{x}^{\#}=0$ so that the above equality simplifies to

$$
\left.\Delta_{S^{1, q}} f\right|_{S^{1, q}}(\mathbf{x})=\left(1-x_{1}^{2}\right) P^{\prime \prime}\left(x_{1}\right)+(1-n) x_{1} P^{\prime}\left(x_{1}\right) .
$$

Combining this with Lemma 2 gives (6).

Corollary 8. For $n \geq 3$, there is (up to scaling) a unique $K$-invariant spherical function of degree $\rho$ given by

$$
\int_{K}(k \mathbf{c} \cdot \mathbf{x})^{\rho} d k
$$

where $\mathbf{c}$ is any non-zero isotropic vector in $\mathbf{R}^{1, q}$.

Proof. The ordinary differential equation (6) is linear of degree 2 with a regular singular point at $x_{1}=1$. The indicial equation at this point is

$$
m(m+(n-1) / 2-1)=0 .
$$


Therefore, it has (up to scaling) at most one solution defined on $[1, \infty)$. This solution is known by Proposition 4.

The classical integral formula due to Laplace for Legendre functions is readily derived from the preceding analysis:

Corollary 9. Every solution of the ordinary differential equation

$$
\left(1-x^{2}\right) P^{\prime \prime}(x)-2 x P^{\prime}(x)+\rho(\rho+1) P(x)=0
$$

that is defined on $[1, \infty)$ is a scalar multiple of

$$
P_{\rho}(x)=\frac{1}{2 \pi} \int_{0}^{2 \pi}\left(x+\sqrt{x^{2}-1} \cos \theta\right)^{\rho} d \theta .
$$

Proof. Evaluate the formula from Corollary 8 taking $q=2, \mathbf{c}=(1,0,-1)$ and $\mathbf{x}=\left(x, 0, \sqrt{x^{2}-1}\right)$.

\section{References}

[1] Sigurdur Helgason, Groups and geometric analysis, Pure and Applied Mathematics, vol. 113, Academic Press Inc., Orlando, FL, 1984, Integral geometry, invariant differential operators, and spherical functions. MR MR754767 (86c:22017)

[2] Elias M. Stein and Guido Weiss, Introduction to Fourier analysis on Euclidean spaces, Princeton University Press, Princeton, N.J., 1971, Princeton Mathematical Series, No. 32. MR MR0304972 (46 \#4102)

[3] E. T. Whittaker and G. N. Watson, A course of modern analysis, Fourth ed., Cambridge University Press, 1927.

The Institute of Mathematical Sciences, CIT campus Taramani, Chennai 600113.

E-mail: amri@imsc.res.in

Chennai Mathematical Institute, Plot H1, SIPCOT IT Park, Padur PO, Siruseri 603103. 